\title{
Onset of hydronephrosis and lower urinary tract symptoms in a previously healthy young man: Phyllodes tumour of the prostate as a potential diagnosis
}

\author{
Matteo Ferrari, MD;* Umberto Capitanio, MD; ${ }^{+}$Nathalie Rizzo, MD; ${ }^{\xi}$ Massimo Freschi, MD; Francesco \\ Montorsi, MD; Patrizio Rigatti, MD*
}

*Advanced Urotechnology Center, "Istituto Auxologico Italiano," Milan, Italy; †'Department of Urology and Urological Research Institute, University Vita-Salute, San Raffaele Hospital, Milan, Italy; ${ }^{\S D e p a r t m e n t ~}$ of Pathology, Scientific Institute San Raffaele Hospital, Milan, Italy

Cite as: Can Urol Assoc J 2014;8(7-8):e561-3. http://dx.doi.org/10.5489/cuaj.1984 Published online August 11, 2014.

\section{Abstract}

We report the case of a 50-year-old healthy man with early onset of micturition symptoms associated with an elevated total prostatespecific antigen. On physical examination, we found an enlarged prostate; a first-line ultrasound of the urinary tract revealed local disease which covered the entire small pelvis. A computed tomography scan confirmed the presence of a $12.5 \times 11.0 \times 9.5-\mathrm{cm}$ multicystic prostatic mass, compressing the bladder and pelvic ureters, associated with right hydronephrosis. Renal function was preserved and prostatic biopsies was negative for malignant disease. The mass was completely removed through transvesical approach and histological analysis diagnosed a low-grade phyllodes tumour of the prostate. The patient was free of local recurrence and metastasis 36 months after surgery.

\section{Introduction}

Phyllodes tumor of the prostate (PTP), also called stromalepithelial cystic tumour, is very rare entity, with under 100 cases described in the literature. According to morphological classification, PTP is one of four histological patterns of stromal tumours of the prostate with uncertain malignant potential (STUMP). PTP is usually diagnosed in young men, with early onset of obstructive urinary symptoms. As shown in this case report, PTP may reach a considerable size, leading to potential damage of the upper urinary tract before being diagnosed.

\section{Case report}

A previously healthy 50-year-old man presented with bladder voiding symptoms associated with an elevated prostate specific antigen (PSA) $(10.25 \mathrm{ng} / \mathrm{mL})$. Endorectal digital examination revealed a prostate significantly increased in size, not aching, with hard-elastic consistency; physical examination did not reveal an abdominal mass.

An initial abdominal ultrasound was followed by a computed tomography scan with contrast medium, showing the presence of $12.5 \times 11.0 \times 9.5$-cm multicystic prostatic mass, mostly solid, capsulated, sparing the most caudal part of the gland and not involving seminal vesicles. The mass occupied almost the entire small pelvis and anteriorly compressed the bladder, posteriorly the sigma and laterally pelvic ureters; this confirmed the presence of a right hydronephrosis of medium grade, causing a right hydronephrosis of medium grade. There was no evidence of abdominal lymphadenopathy (Fig. 1).

The sieric creatinine level was $1.2 \mathrm{mg} / \mathrm{dL}$. Extended prostatic sampling (24 cores) was negative for any neoplasm. The staging was complete with a chest $x$-ray that did not show lesions in the lung parenchyma.

The patient underwent complete transvesical prostatesparing excision of the mass, through soprapubic laparotomy incision according to Pfannenstiel. Postoperatively, the patient's creatinine level was down to $1.01 \mathrm{mg} / \mathrm{dL}$ and total PSA to $0.5 \mathrm{ng} / \mathrm{mL}$.

Macroscopically, the lesion was of multicystic appearance, irregular, gray-white, brittle shear. The microscopical examination (Fig. 2) revealed a cystic neoplasm composed of stromal and epithelial stromal papillae and solid areas.

The first pattern was a hypercellular stroma with scattered stromal cells containing pleomorphic and degenerative-appearing nuclei admixed with benign prostatic gland. The second pattern was a hypercellular stroma composed of bland spindle cells with eosinophilic cytoplasm. Stromal cells resulted with mild atypia, moderately positive for actin and desmin. Leaf-like hypocellular fibrous stroma covered by benign-appearing prostatic epithelium formed the phyllodes pattern of STUMP. The epithelium of the papillae appeared cylindrical, PSA positive, with foci of squamous 


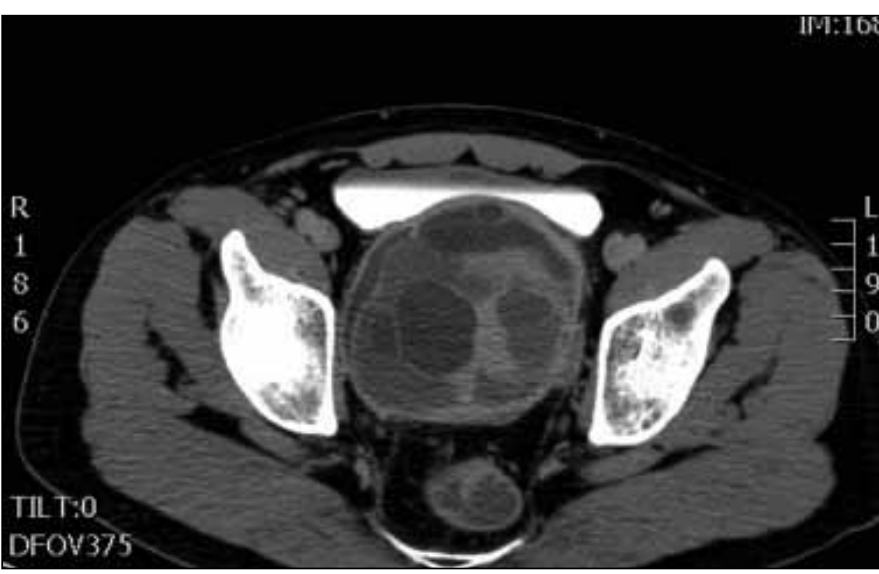

Fig. 1a. Axial computed tomography image showing the large prostatic mass with cystic components that compresses the bladder.

metaplasia, transitional atypia and rare mitosis (mitotic rate $1 \times 10$ high-powered field), in the absence of necrosis. The definitive histologic diagnosis was low-grade stromal-epithelial PTP. ${ }^{1}$ The patient was free of local recurrence and metastasis 36 months after surgery.

\section{Discussion}

The clinical characteristics of PTP in our case were less common than in most cases previously described. Most of the histological diagnoses of PTP refer to surgical specimens of patients with obstructive urinary symptoms undergoing transurethral resection of the prostate. ${ }^{2}$ This data may be explained by the fact that histological diagnosis by biopsy is difficult and the PTP usually arises in the central zone of the prostate near the verumontanum. In the largest available series, specimen weight ranged from 3.5 to $51 \mathrm{~g}^{2}{ }^{2}$ however, this tumour can arise in the peripheral zone and its dimension can be very different.

Aguilar and colleagues ${ }^{3}$ reported a case of a 67 -year-old patient with hematuria secondary to a $900-\mathrm{mL}$ cystic PTP starting from the left base of the prostate. In another case, Chung and colleagues ${ }^{4}$ found a cystic PTP of $13.5 \mathrm{~cm}$ in diameter weighing $300 \mathrm{~g}$, occupying the whole pelvis and palpable as a soft abdominal mass in a 65-year-old patient. Kawamorita and colleagues ${ }^{5}$ described the most striking case - a 55-year-old patient with dysuria affected by a PTP of $60 \mathrm{~cm}$ in diameter weighing $8600 \mathrm{~g}$, extending into the retroperitoneum and compressing the bladder, bowel, liver and kidneys.

The PTP diagnosed in our patient was significant in size and compressed neighbouring pelvic organs with serious potential consequences. Although a rare prostatic cause of hydronephrosis, PTP shows that early symptoms of lower urinary tract in a young man may portend extended disease.

Regarding the search of clinical markers, although PSA is

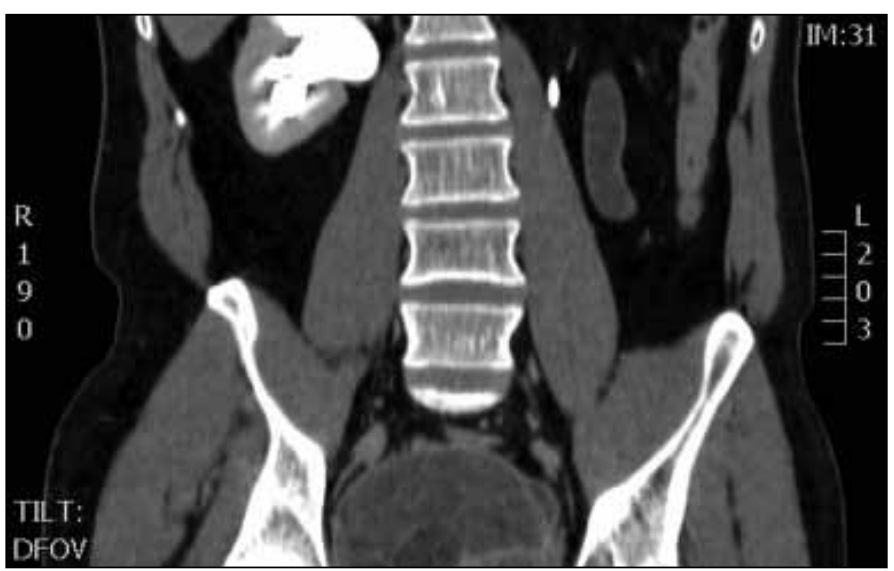

Fig. 1b. Coronal computed tomography (CT) image showing right hydronephrosis.

expressed in the epithelium of PTP often resulting in a value higher than normal in these patients, a correlation between total PSA value and tumour size or grading score is not present in the literature. Nagar and colleagues ${ }^{6}$ studying epithelial proliferations found that in STUMPs with a phyllodes pattern, there is a tendency toward urothelial and squamous metaplasia; this data were confirmed in the histopathologic analysis of our specimen.

In this context, it has been reported that PTP usually becomes malignant for stromal overgrowth and sarcomatous transformation. ${ }^{7}$ To better study the outcome, Bostwick and colleagues $^{2}$ introduced a grading system for PTP based on 5 histologic features (stromal cellularity, cytological atypia, mitotic number of figures, stroma-to-epithelium ratio and necrosis) determining a low, intermediate and high grade. They found a trend towards a higher incidence of recurrence and decreased survival in high- compared to lowgrade tumours. Specifically, even if low-grade tumours are subject to a high percentage of recurrence $(50 \%)$ and progression, malignancy occurs after several relapses. In this series, a low-grade sarcoma with distant metastasis emerged in 1 of 14 low-grade patients after 14 years from their initial diagnosis and after 5 episodes of recurrence. Lastly, a correlation between the size of PTP and its grading score was not identified; in our case, the final pathology was diagnosed as a low-grade PTP, as reported by Aguilar and colleagues ${ }^{3}$ and Chung and colleagues. ${ }^{4}$

\section{Conclusion}

With the limit of the length of our follow-up, in agreement with previous cases of giant PTP,3,5 we confirm that total resection, with an organ-sparing approach may be an appropriate initial surgical treatment in the presence of a lowgrade tumour. A close follow-up regimen is necessary to identify early signs of recurrence and progression. 


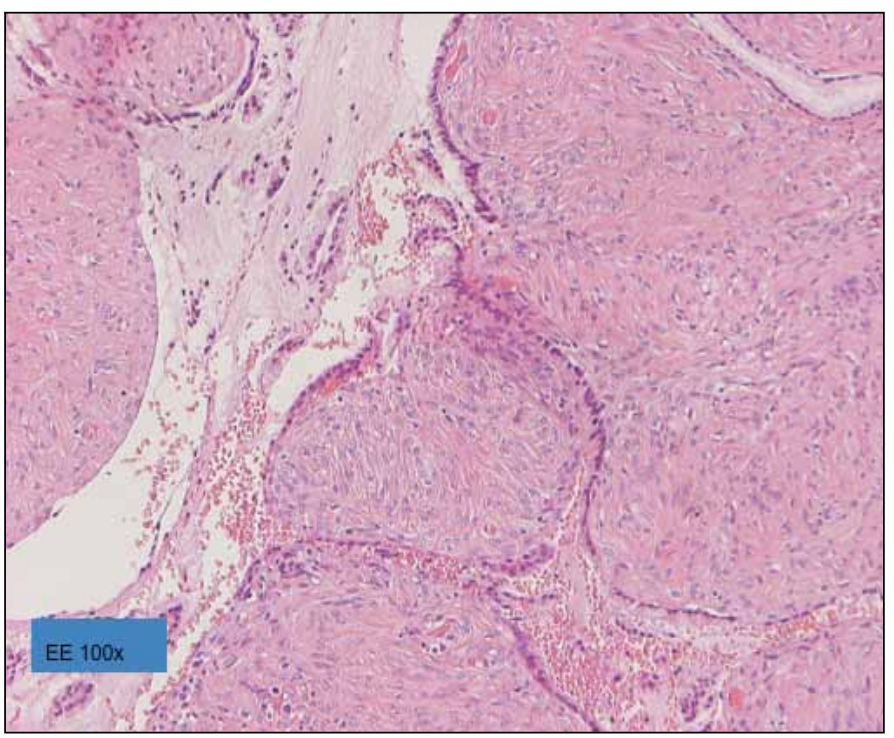

Fig. 2a. Microscopic images demonstrating the epithelial and stromal components of the cystic tumour.

Competing interests: Dr. Ferrari, Dr. Capitanio, Dr. Rizzo, Dr. Freschi, Prof. Montorsi and Prof. Rigatti all declare no competing financial or personal interests.

This paper has been peer-reviewed.

\section{References}

1. Epstein II, Cubilla AL, Peter Ah. Tumors of the prostate gland, seminal vescicles, penis and scrotum. AFIP Atlas of Tumor Pathology (series 4), ARP Press; 2011.

2. Bostwick DG, Hossain D, Qian J, et al. Phyllodes tumor of the prostate: long-term follow up study of 23 cases. J Urol 2004;172:894-9. http://dx.doi.org/10.1097/01.ju.0000134580.71261.57

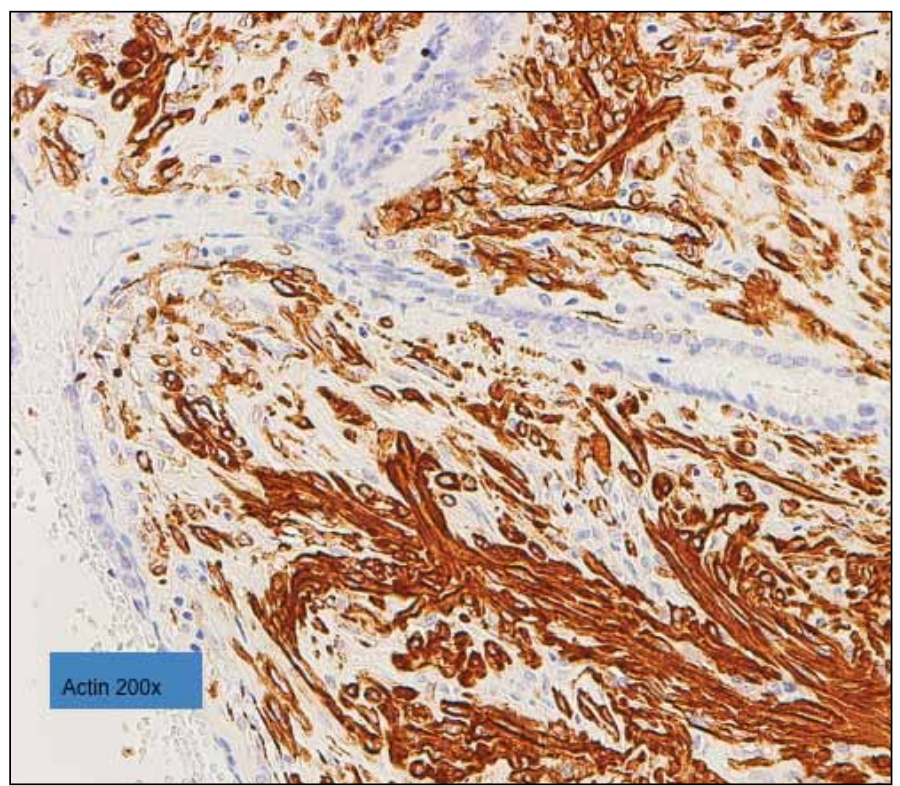

Fig. $\mathbf{2 b}$. Microscopic images demonstrating the actin-positivity of stromal cells.

3. Aguilar B, Villalobos M, Sotomayor M. Phyllodes tumor of the prostate. Urol 2009;73:272. http:// dx.doi.org/10.1016/i.urology.2008.08.466

4. Chung HC, Lee HS, Kim TI, et al. A large cystic phyllodes tumor of the prostate. Yonsei Med J 2009;50:1746. http://dx.doi.org/10.3349/ymi.2009.50.1.174

5. Kawamorita N, Inaba Y, Soma F, et al. Giant phyllodes tumor of the prostate. Nihon Hinyokika Gakkai Zasshi 2007;98:781-5.

6. Nagar $M$, Epstein Jl. Epithelial proliferations in prostatic stromal tumors of uncertain malignant potential (STUMP). Am J Surg Pathol 2011;35:898-903. http://dx.doi.org/10.1097/PAS.0b013e318214f2f2

7. Lopez-Beltran A, Gaeta JF, Huben R, et al. Malignant phyllodes tumor of prostate. Urol 1990;35:164. http://dx.doi.org/10.1016/0090-4295(90)80068-X

Correspondence: Dr. Matteo Ferrari, Advanced Urotechnology Center, "Istituto Auxologico Italiano," via Mercalli 30, 20122 Milan, Italy; em.ferrari@alice.it 